\title{
Repelente de insectos voladores a base de aceite esencial de mastranto (hyptis suaveolens)
}

\author{
Flying insect repellent based on mastranto essential oil (hyptis \\ suaveolens) \\ Nohely Ostos \\ nohelyostos@gmail.com \\ María Rodríguez \\ rodgo3112@gmail.com \\ Universidad de Carabobo, Venezuela
}

\section{RESUMEN}

Investigación centrada en la elaboración de una crema repelente de insectos voladores a base de aceite esencial de mastranto, extraído por arrastre con vapor en una planta tipo banco y por destilación-extracción simultánea en un equipo Likens-Nickerson. Se realiza un diseño factorial para la evaluación de cada método. Variables escogidas: la cantidad de carga alimentada al sistema y el flujo de vapor $y$, por medio de un análisis de varianza, se encuentra que el mayor rendimiento fue de $0,31 \%$ para $300 \mathrm{~g}$ de hojas secas con un flujo de $831,018 \mathrm{~mL} / \mathrm{h}$. En la extracción destilación simultánea, las variables analizadas son tamaño de material vegetal y cantidad de material alimentado, obteniéndose un rendimiento máximo de $0,4 \%$ para $20 \mathrm{~g}$ de hojas secas con un tamaño de 2-4 mm. Del análisis cromatográfico se encontraron Lfenchona y eucaliptol, a este último se le responsabiliza por el efecto repelente. La evaluación dermatológica concluye que todos los voluntarios de la prueba no presentaron efectos no deseados $y$ aceptación del producto.

Palabras clave: Aceite esencial de mastranto; arrastre con vapor en una planta tipo banco; crema repelente; destilación-extracción simultánea; diseño factorial; equipo Likens-Nickerson; hyptis suaveolens

\begin{abstract}
Research focused on the preparation of a flying insect repellent cream based on mastranto essential oil, extracted by steam dragging on a bench-type plant and by simultaneous distillation-extraction in a Likens-Nickerson equipment. A factorial design is made for the evaluation of each method. Variables chosen: the amount of load fed to the system and the steam flow and, through an analysis of variance, it is found that the highest yield was $0.31 \%$ for $300 \mathrm{~g}$ of dry leaves with a flow of $831,018 \mathrm{~mL} / \mathrm{h}$. In the simultaneous distillation extraction, the variables analyzed are plant material size and quantity of material fed, obtaining a maximum yield of $0.4 \%$ for $20 \mathrm{~g}$ of dried leaves with a size of $2-4 \mathrm{~mm}$. From the chromatographic analysis, L-fenchone and eucalyptol were found, the latter being responsible for the repellent effect. The dermatological evaluation concludes that all the volunteers of the test did not present unwanted effects and acceptance of the product.
\end{abstract}

Key words: Essential oil of mastranto; characterization; repellent cream; factorial design 
INTRODUCCIÓN

El mastranto o hyptis suaveolens es una maleza anual, originaria de América Pérez et al., (2011). Es de la familia de la menta y crece en rastrojos y orillas de caminos de las tierras cálidas y templadas, con tallos espinosos y hojas opuestas, aromáticas, ásperas y de borde aserrado. Su capacidad para adaptarse a ambientes que van desde el nivel del mar hasta los $900 \mathrm{msnm}$, lo convierten en una fuente potencial de aceites esenciales Peerzada, (1997); Chacín et al. (2004). Ésta tiene una alta capacidad de producción de aromas, lo cual se manifiesta en el olor que se percibe en sus matorrales, en este sentido, se utiliza en diferentes áreas de la medicina convencional. El extracto de la planta entera (sin raíz) se usa en la medicina tradicional como un excelente hipotensor (Chacín et al., 2004; Messias et al., 2015). Adicionalmente tiene propiedades desinfectantes y astringentes, es tónico del estómago, regulador del sistema circulatorio y se usa además contra los insectos y parásitos. Los principios activos de esta planta son el mentol, mentona y carvona (Álvarez y Medina, 2008).

Los aceites esenciales son básicamente extractos de flores, plantas, semillas, hojas, tallos, cortezas y frutos. Éstos son obtenidos de la flora y son mezclas de aproximadamente 100 componentes, los cuales los convierten en una fuente potencial, su composición depende de la localidad y del método de extracción utilizado (Nannapaneni et al., 2009; Soto et al., 2013). Para extraerlo de una planta, pueden emplearse diferentes métodos, entre ellos destacan la hidrodestilación, extracción con fluidos supercríticos y extracción por hidrodifusión. La producción de aceites esenciales derivados de la flora aromática constituye una base importante en varias ramas industriales.

Actualmente, existen una cantidad de enfermedades de diferentes tipos, las cuales han venido modificando sus variantes a través del tiempo, donde las transmitidas por los insectos siempre estarán presentes. Un ejemplo de este tipo de insectos son las moscas, mosquitos y abejas (Daza y Flores, 2006). La mayoría de las sustancias sintetizadas para crear una barrera entre humanos e insectos incluyen en su formulación productos que pueden ser nocivos para la salud, como es el caso del $\mathrm{N}, \mathrm{N}$-dietil-toluamida (DEET, por sus siglas en inglés) y el Bayrepel o picaridina (ácido 1-piperidincarboxílico, 2(2-hidroxietil)-1-metilpropiléster) los cuales, uno en mayor proporción que el otro, son tóxicos para el sistema nervioso central, según (Corbel et al., 2009; Ranson et al., 2011)

En este sentido, es importante el desarrollo de productos para el control de insectos que permitan reemplazar los repelentes sintéticos, que ofrezcan mayor seguridad para el ambiente y ahuyenten este tipo de agentes causantes de enfermedades. La principal característica es la presencia de un fuerte aroma no tolerable para los insectos, existen en la naturaleza compuestos que cumplen con estas propiedades, como lo son los aceites esenciales, los cuales están formados por varias sustancias orgánicas volátiles, que pueden ser alcoholes, cetonas, ésteres y aldehídos y que se producen y almacenan en los canales secretores de las plantas. En este sentido, se requiere conocer el método de extracción que garantice una 
mejor calidad en cuanto a la composición del aceite $\mathrm{y}$, por tanto, un mejor aprovechamiento de sus propiedades para fines específicos. Para esto se emplean dos métodos de extracción, posteriormente se realiza un análisis de la composición y su grado de pureza así como de sus propiedades repelentes. Adicionalmente, se utilizarán aceites esenciales de eucalipto (eucalyptus citriodora) y mandarina (eitrus nobilis) para de esta manera proporcionarle al producto final, características olfativas diferentes y más agradables que las que se le atribuyen al mastranto (Singh et al., 2012; Khalid, et al., 2012).

Son pocos los estudios que se han realizado sobre el aceite esencial de mastranto y su proceso de extracción. No obstante, se encuentra el de Dutta et al. (2009) quienes realizan una investigación sobre las actividades repelentes e insecticidas de $H$. suaveolens (Lamiaceae) del aceite esencial extraído de sus hojas contra cuatro (4) plagas de coleópteros de grano almacenado de productos almacenados, el Callosobruchus maculatus (Bruchidae), Rhyzopertha dominica (Bostrychidae), Sitophilusoryzae (Curculionidae) y Triboliumcastaneum (Tenebrionidae). Estos insectos atacan a los granos almacenados y no a insectos voladores que pueden ser considerados transmisores de enfermedades a los seres humanos.

Abagli y Alavo (2011) realizaron una investigación sobre la eficacia del aceite esencial de menta Bush, $H$. suaveolens. En este se muestra la iniciativa de promover un estudio para evaluar el efecto repelente del $H$. suaveolens por medio de la destilación al vapor y el aceite se aplica directamente sobre la piel. Por su parte, Conti et al., (2012) evaluaron la actividad larvicida y repelente del aceite esencial de $H$. suaveolens (Lamiaceae) sobre el mosquito Aedes albopictus Skuse (Diptera: Culicidae). En esta investigación, el aceite esencial extraído de hojas frescas de $H$. suaveolens (Lamiaceae) y sus principales componentes fueron evaluados contra el mosquito tigre asiático A. albopictus (Diptera: Culicidae), actualmente el mosquito más invasor en todo el mundo. El aceite esencial de $H$. suaveolens tuvo actividad insecticida contra las larvas de $A$. albopictus y la mortalidad dependió de la dosis.

En el siguiente trabajo se busca aprovechar el mastranto, se extrajo su aceite esencial empleando los métodos de arrastre con vapor y extracción por destilación simultánea, aplicando análisis de varianza estadístico para establecer las diferencias entre los rendimientos de los diferentes extractos obtenidos. Seguidamente se caracteriza para conocer los componentes aromáticos, plantear y preparar formulaciones a diferentes concentraciones, se emplearon pruebas para verificar su estabilidad física y química, y garantizar la calidad del producto. Finalmente, se ejecutaron ensayos dermatológicos, microbiológicos y de repelencia con el fin de desarrollar un repelente natural enfocado a los insectos voladores empleando el aceite esencial de mastranto obtenido como ingrediente principal, resaltando sus propiedades medicinales y los beneficios que puede brindar, presentándose como una alternativa ante los repelentes sintéticos que abastan al mercado 
MATERIALES Y MÉTODOS

\section{Preparación de las muestras}

Para llevar a cabo las corridas experimentales es necesaria la preparación del material vegetal a emplear. Una vez realizada la recolección se cortan los tallos dejando sólo las hojas, ya que es donde está contenida la mayor cantidad de aceite esencial (Chacín et al., 2004). Para eliminar la humedad de las hojas se sometieron las mismas a una etapa de secado, es decir, colocarlas sobre hojas de papel absorbente con incidencia directa del Sol, por un período de cuatro días aproximadamente. Posteriormente, se trituran para permitir una mejor área de contacto con el agua, que servirá como medio para la realización de la extracción.

\section{Variables a manipular}

Para establecer las condiciones de operación que permitieran alcanzar el mayor rendimiento, se consultaron investigaciones donde se emplearon los mismos métodos de extracción, en este sentido las variables analizadas son tamaño de material vegetal y cantidad de material alimentado. En el caso de la destilación por arrastre con vapor usando la planta tipo banco, se escoge tamaño de material vegetal y cantidad de material alimentado.

\section{Selección del diseño experimental}

El diseño factorial se hará a dos (2) niveles alto $(+)$ y bajo (-). Para ambos métodos, utilizando las variables o factores establecidos en cada uno (Prat y TortMartorell, 2004). En la determinación de los rangos de operación se realizaron extracciones preliminares donde se evalúa la influencia de diferentes valores para cada factor establecido sobre el rendimiento obtenido, según lo reportado por (Pérez et al., 2011; Álvarez y Medina, 2008). Una vez que se estipularon los rangos a utilizar se definen las variables de respuesta y factores. En este caso ya se ha determinado previamente la variable de respuesta y los factores influyentes, se aleatoriza la toma de datos, es decir se estudia la combinación de los factores a distintos niveles, llevando a cabo las diferentes extracciones calculando los efectos mediante algoritmo de signos para finalmente concluir acerca de los efectos de las variables y su comportamiento (Prat y Tort-Martorell, 2004).

\section{Selección de los parámetros operacionales para la obtención de aceite esencial de mastranto $(\mathrm{H}$. suaveolens) mediante una planta tipo banco}

El equipo está formado de un reactor o alambique, donde se coloca el material a destilar. Este consiste en que de un tanque cilíndrico con una tapa con cierre hermético; del centro de la tapa, sale un tubo conductor de los vapores hacia el condensador. En su fondo se encuentra la generación de vapor, además se halla una válvula de drenaje para permitir que el agua alimentada o condensada sea descargada fácilmente al final de la extracción. También consta de un separador que se encarga de separar el aceite esencial obtenido del hidrolato, que es el agua residual que se forma por condensación del vapor que ha atravesado la materia vegetal durante el proceso de obtención del aceite esencial (Ostos y Rodríguez, 2016). En primer lugar, se pesa 
la materia prima fresca, secada y cortada en trozos de un tamaño determinado, colocándolo en capas sobre rejillas metálica dentro de una cesta. Se agrega agua hasta donde indica el nivel en el $\begin{array}{llll}\text { reactor } & \text { (aproximadamente } & 7 & \text { L) }\end{array}$ garantizando que la resistencia de calentamiento quedara sumergida, se introdujo la cesta que contiene la muestra y se coloca la tapa del reactor, asegurando su cierre hermético.

Se realiza el montaje del equipo de extracción de arrastre con vapor a nivel piloto, registrando la temperatura inicial tanto en el reactor, como en el agua de enfriamiento que circula por el condensador; considerando que se enciende con anterioridad. Se ajustó con pinzas el vaso florentino, de tal manera que soporte el peso de la mezcla aguaaceite condensada. Se enciende y se deja calentar hasta ebullición que dio inicio a la obtención de la primera gota de destilado anotando el tiempo en que se recogió y la temperatura de los vapores en el tope del recipiente contenedor. Una vez iniciada la condensación y pasado el tiempo de extracción establecido en el diseño experimental, se dejó reposar durante 10 min la mezcla agua-aceite recolectada en el vaso florentino para que ocurriera la separación del agua con el aceite. Se descargó por la válvula de descarga del vaso florentino, gota a gota, para asegurar que el aceite esencial no se quede adherido en la superficie del vaso recolector. La parte orgánica, se introdujo en un envase recolector donde se le añadió sulfato de sodio anhídrido para eliminar el remanente de agua que quedó luego de la decantación, el aceite extraído fue trasvasado a un envase que había sido prepesado, agregando un cristal de butilhidroxitolueno para evitar la oxidación del mismo. Finalmente fue pesado el envase recolecto con el aceite (Escobar, 2012; Alcántara, 2015).

\section{Selección de parámetros opera- cionales para la extracción de aceite esencial de mastranto ( $H$. suaveolens) empleando el método de destilación y extracción simultánea (EDS).}

Para el presente estudio se modifica el cuerpo de extracción del equipo LikensNickerson (Escobar, 2012) con la finalidad de facilitar la recolección del aceite esencial luego de concluido el proceso de destilación y extracción simultánea. Ésta modificación consiste en incorporarle una válvula de seguridad en la parte inferior de la curva donde ocurre la separación de las fases, aprovechando de esta manera la diferencia en cuanto a densidades $y$ facilitando la recuperación del extracto.

Inicialmente, se pesa el material vegetal previamente picado, se instala el cuerpo de extracción- destilación junto con el dedo frío en el soporte universal y se incorpora el baño refrigerante circulante. Se agrega el volumen de agua destilada y el material vegetal en un balón de $1000 \mathrm{~mL}$ y diclorometano en el balón de $250 \mathrm{~mL}$. Al tubo U se agregó diclorometano $(25 \mathrm{~mL})$ completando con agua destilada para lograr la interface hasta aproximadamente un (1) $\mathrm{cm}$ antes del inicio de la zona de condensación, se incorporaron los balones al montaje colocando bajo estos el equipo de calefacción correspondiente. Se enciende el baño refrigerante circulante para asegurar su enfriamiento y la buena circulación alrededor del dedo frío manteniendo una temperatura alrededor 
de los dos (2) ${ }^{\circ} \mathrm{C}$ asegurando así la condensación del disolvente. Una vez encendida la manta y la plancha de calentamiento para el balón con material vegetal y para el baño del solvente respectivamente, se lleva el registro de temperatura, tomando el tiempo desde la caída de la primera gota de condensado, transcurrido el tiempo de extracción se deja enfriar el equipo de 15 a 20 minutos. Se retira el extracto, la fase orgánica contenida en la " $\mathrm{U}$ " del equipo, deshidratándose con $\quad \mathrm{Na}_{2} \mathrm{SO}_{4} \quad$ y concentrándose por rotaevaporación. Finalmente, se transfiere el aceite esencial obtenido en un envase ámbar limpio para conocer el volumen recolectado y se añade un pequeño cristal de butilhidroxitolueno (BHT) para evitar la oxidación y se reserva en un espacio refrigerado.

\section{RESULTADOS}

La formulación de la crema repelente consiste en una emulsión de aceite en agua $(0 / W)$. Ésta se realiza a través del análisis de la proporción de aceite adecuada para una base previamente seleccionada, caracterizándola para, posteriormente, realizarle pruebas de estabilidad $y$ microbiológicas. En las tablas 1 y 2 se muestran la composición y propiedades físicas para realizar $2.000 \mathrm{~g}$ de la crema base.

Tabla 1. Fórmula cuantitativa de la crema base utilizada en la fórmula de repelente

\begin{tabular}{c|l|c|c}
\hline No. & \multicolumn{1}{|c|}{ Materias primas } & $\mathbf{\%} \mathbf{p / p}$ & Función \\
\hline & \multicolumn{1}{|c}{ Fase A (Acuosa) } & & \\
\hline 1 & Agua Desionizada & 75,150 & Vehículo \\
2 & Carbomer & 0,400 & Espesante \\
3 & Glycerina & 5,000 & Agente Humectante \\
4 & PropylParasept & 0,150 & Preservante \\
5 & MethylParasept & 0,350 & Preservante \\
6 & DMDM Hydantoin & 0,400 & Preservante \\
7 & CetearylAlcoholCeteareht -20 & 2,000 & Emulsificante \\
8 & Polisurbato-60 & 1,000 & Emulsificante \\
5 & Propilen Glicol & 2,000 & Agente Humectante \\
\hline & \multicolumn{1}{|c}{ Fase B (Oleosa) } & 2,000 & \\
\hline 6 & CetearylAlcoholCeteareht -20 & 4,000 & Emulsificante \\
7 & Cetearyl Alcohol & 3,000 & Espesante \\
8 & Aceite Mineral & 2,000 & Emoliente \\
9 & Ciclomethicona & 1,500 & Emoliente \\
\cline { 2 - 4 } 10 & SorbitanSesquiolato & & Emulsificante \\
\hline \multirow{2}{*}{11} & Fase C (Neutralización/Gelificación) & 0,750 & Neutralizante \\
\cline { 2 - 4 } 12 & Trietanolamina & 0,300 & Fragancia \\
\hline
\end{tabular}


Tabla 2. Propiedades físicas de la crema repelente

\begin{tabular}{c|c}
\hline Propiedades Físicas & Descripción \\
\hline Color & Blanco \\
Olor & Mandarina dulce \\
Aspecto & Homogéneo \\
Textura & Suave, cremosa \\
Extensibilidad & Buena \\
\hline
\end{tabular}

Obtención de aceite esencial de mastranto (H. suaveolens) mediante una planta tipo banco

Ostos y Rodríguez (2016) y BoschFusté et al (2007) reportan un tiempo de extracción no mayor a 60 minutos, puesto que luego de transcurrido este tiempo, no se obtiene una diferencia relevante sobre el rendimiento. Se decide tomar en consideración las mejores combinaciones experimentales reportadas por Bosh-Fuste et al. (2007) bajo las mismas condiciones de operación (300 y 400 gr durante 1 h), a fin de evaluar la influencia que pueda tener este factor sobre el rendimiento. Posterior al barrido con los diferentes flujos de vapor de arrastre, se establecieron como valores para las pruebas experimentales los niveles 7 y 9 del reóstato correspondiente a 619,85 y $831,02 \mathrm{~mL} / \mathrm{h}$, respectivamente, los cuales se consideraron como flujos mínimos óptimos en el alambique (sistema de destilación). La Tabla 3 muestra el rendimiento obtenido en la extracción por arrastre con vapor del aceite de mastranto para la mejor combinación de variables.

Tabla 3. Rendimiento obtenido en la extracción por arrastre con vapor del aceite de mastranto para la mejor combinación de variables

\begin{tabular}{|c|c|c|c|c|c|}
\hline $\begin{array}{l}\text { Masa inicial de } \\
\text { la muestra } \\
\text { (mi } \pm \text { ) g }\end{array}$ & $\begin{array}{l}\text { flujo de vapor } \\
(\mathrm{Mv} \pm) \mathrm{mL} / \mathrm{h}\end{array}$ & $\begin{array}{c}\text { Masa del } \\
\text { recolector } \\
\text { vacío } \\
(\mathrm{mv} \pm) \mathrm{g}\end{array}$ & $\begin{array}{c}\text { Masa del } \\
\text { recolector } \\
\text { lleno } \\
(\mathrm{mll} \pm) \mathrm{g}\end{array}$ & $\begin{array}{l}\text { Masa de } \\
\text { extracto } \\
\text { obtenido } \\
(\mathrm{me} \pm) \mathrm{g}\end{array}$ & $\begin{array}{c}\text { Porcentaje de } \\
\text { rendimiento } \\
\text { (\%) Adim }\end{array}$ \\
\hline 300,011 & 831,018 & 8,055 & 8,998 & 0,943 & 0,314 \\
\hline 300,010 & 831,018 & 8,090 & 9,012 & 0,922 & 0,307 \\
\hline
\end{tabular}

Obtención de aceite esencial de mastranto (H. suaveolens) mediante destilación-extracción simultánea

La destilación-extracción simultánea es una técnica donde se retiran de la muestra los analitos del aroma por medio de vapor de agua. Estos son transferidos a la fase orgánica cuando ocurre la condensación simultánea de los líquidos, la fase acuosa y la orgánica son separadas en el cuerpo de extracción y cada una regresa a su recipiente por lo que se da un reflujo continuo. Para la selección del solvente y la cantidad a emplear se recomiendan que el punto de ebullición sea lo más bajo posible para evitar problemas con los volátiles ya extraídos, los cuales son sensibles a cambios de temperatura elevados 
(Guayadol, 1994). Se trabaja con una disposición para solventes más denso que el agua y se prefieren los hidrocarburos alifáticos, alifáticos halogenados, alicíclicos, aromáticos (Castellanos, 2014). En este sentido, se selecciona el diclorometano por su bajo punto de ebullición, con un volumen de $50 \mathrm{~mL}$, divididos entre el cuerpo extractor o " $U$ " $(20 \mathrm{~mL})$ y el balón de recolección $(30 \mathrm{~mL})$.

\section{Análisis de diferentes formulaciones de repelentes de insectos voladores usando el aceite esencial de mastranto (H. suaveolens)}

La formulación del repelente se hizo a nivel laboratorio, se siguieron las indicaciones de que para productos cosméticos solo se debe tomar entre a $1 \mathrm{a}$ $10 \%$ de aceite esencial, es por esto que se plantearon como posibles concentraciones de 1,5 y $7 \%$ de aceite esencial. Para evaluar la estabilidad del producto, se utilizaron muestras de cinco (5) $\mathrm{mL}$, las cuales se sometieron a distintos grados de temperaturas $\quad\left(5^{\circ} \mathrm{C}\right.$, aproximadamente $40^{\circ} \mathrm{C}$ y ambiente). En este sentido, la Figura 1 muestra el comportamiento del $\mathrm{pH}$ en las diferentes formulaciones sometidas al estudio de estabilidad preliminar. Adicionalmente, una muestra de cada formulación fue sometida a 3000 rpm por 30 minutos, al finalizar el proceso se observa que las muestras de 1 y $5 \%$ de aceite esencial no tuvieron un cambio aparente en su aspecto y se mantuvieron homogéneas, por su parte la muestra de $7 \%$ presenta una separación de fases como puede observarse en la Figura 1, lo cual indica que la crema en esa composición es inestable y la misma debe ser ajustada o rechazada.

De lo expuesto anteriormente se tiene que en cuanto a estabilidad las composiciones más factibles fueron las de 1 y 5\% con las cuales se evaluó posteriormente la eficacia del repelente, siendo la formulación de $7 \%$ de aceite esencial descartada.

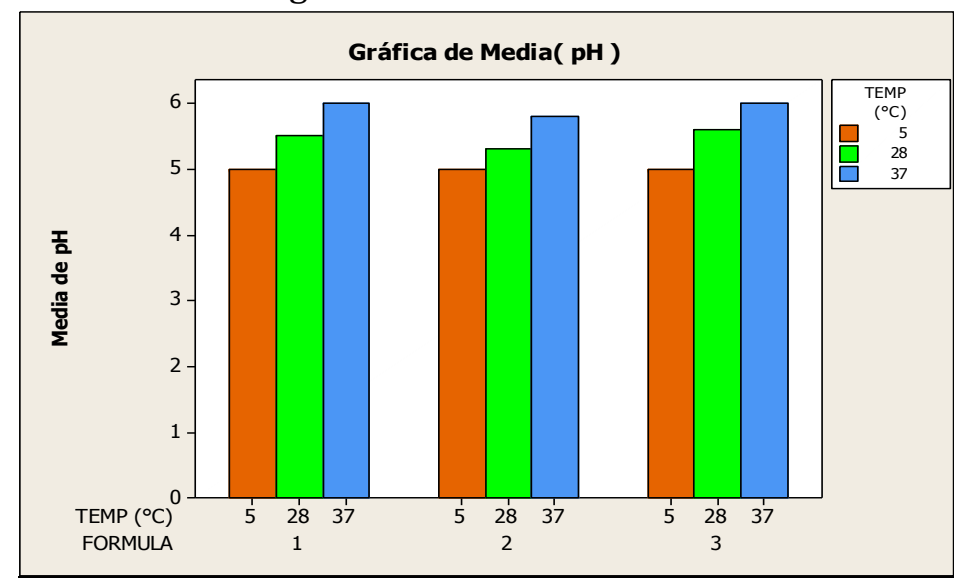

Figura 1. Comportamiento del $\mathrm{pH}$ en las diferentes formulaciones sometidas al estudio de estabilidad preliminar 
Para el análisis microbiológico se realiza una siembra "presencia-ausencia" donde se utilizaron tres (3) medios de cultivos diferentes para tener una visión del tipo de microorganismos presentes en la crema y que pudiesen crecer en las condiciones adecuadas Para esto se usaron tres (3) tipos de medios de cultivos PDA (agar-papa-dextrosa) que permite el crecimiento de hongos y levaduras, TGEA para bacterias y aerobios mesófilos y WL (Wallerstein) para bacterias, hongos, levaduras $\mathrm{y}$ termófilos aerobios. Se sembraron las muestras por duplicado, en forma estriada y se dejaron las muestras a $28^{\circ} \mathrm{C}$ por cuatro días. Al finalizar el plazo de incubación no se visualizaba crecimiento en la siembra en PDA ni en la de TGEA, no se observa la presencia de mohos ni levaduras. Con el WL existe una tendencia diferente; al ser este medio uno de crecimiento mixto, se realizó una dilución, filtración y posterior siembra en TGEA de la crema repelente, siguiendo el procedimiento descrito en el capítulo anterior.

Cuando el número de microorganismos presentes en una muestra tiende a ser bajo, se recurre a su filtración a través de membrana que retienen bacterias, luego la membrana es colocada sobre un medio agarizado y se procede a su incubación. Una vez culminado el proceso de incubación se realizó el conteo de microorganismos presentes. El recuento de microorganismos viables en placa se basa en la formación de una colonia a partir de cada célula viable, utilizando como soporte, medios agarizados en placas de Petri. Como no es posible asegurar que toda colonia derive de un solo microorganismo, la forma correcta de expresar los resultados es como unidades formadoras de colonias (UFC). Los resultados se muestran en la Tabla 4. Las pruebas dieron como resultado ausencia de mohos y levaduras y unidades formadoras de colonias (UFC) de aerobios mesófilos dentro de los límites estipulados por la norma (ISO,2006), por lo que puede considerársele un producto libre de microorganismos que puedan ser causantes de su deterioro, así como un riesgo de infección al usuario.

Tabla 4. Resultados de los análisis microbiológicos realizados a la crema repelente

\begin{tabular}{c|c|c}
\hline $\begin{array}{c}\text { Microrganismos aerobios } \\
\text { mesófilos totales. } \\
\text { ( UFC/g) }\end{array}$ & $\begin{array}{c}\text { Mohos } \\
\text { (UFC/g) }\end{array}$ & $\begin{array}{c}\text { Levaduras } \\
\text { (UFC/g) }\end{array}$ \\
\hline $4 \times 10^{2}$ & Ausentes & Ausentes \\
\hline
\end{tabular}

\section{Pruebas dermatológicas}

Las pruebas usadas para analizar cosméticos y determinar la sensibilidad y efecto en la piel son mediante parche, donde el aspecto evaluado es por medio de la irritación dérmica. Se escogieron personas voluntarias para determinar si producían reacciones alérgicas, en la prueba señalada no se observaron presencia de alguna reacción al cabo de 24 horas por lo que se procede a repetirla, arrojando el mismo resultado, de manera que es permitida la aplicación del producto y se procede a la siguiente prueba. 


\section{Prueba de repelencia y aceptación al producto final}

Se desarrollaron dos formulaciones y se lleva a cabo la realización de una encuesta a una población de 15 personas en la comunidad Los Manires, ubicada en el municipio Nirgua, estado Yaracuy (Ostos y Rodríguez, 2006), empleando las preguntas claves que ayudarían a evaluar el grado de repelencia, tomando en cuenta las opiniones de los encuestados para elaborar y decidir la mejor formulación, considerando la sensación del producto al aplicarlo sobre la piel, su olor, así como también algunas molestias causadas por los insectos, entre otros. Finalmente, los resultados sobre los efectos no deseados fueron los mismos para las formulaciones. Todas las personas encuestadas están de acuerdo con que el repelente natural no causa efecto irritante sobre la piel al usuario, lo que sustenta el resultado de las pruebas dermatológicas.

\section{CONCLUSIONES}

- Los parámetros operacionales para obtener un mayor rendimiento en la planta tipo banco por arrastre con vapor son cantidad de material vegetal $300 \mathrm{~g}$ y un flujo de vapor de agua igual a 831,018 mL/h. Adicionalmente, los de destilaciónextracción simultánea en el equipo Likens-Nickerson son una carga de material vegetal de $20 \mathrm{~g}$ de hojas secas con un tamaño de 2 a $4 \mathrm{~mm}$.

- El aceite esencial de mastranto presenta una coloración amarillo verdoso, con un olor penetrante mentolado, algo característico del tipo de planta. Los compuestos mayoritarios en la extracción por arrastre con vapor fueron: L-fenchona, eucaliptol y espatulenol, 24,56, 15,13 y $7,14 \%$ respectivamente.

- Los compuestos mayoritarios en la extracción-destilación simultánea fueron: L-fenchona y eucaliptol 17,68 y 7,63\% de porcentaje de área bajo la curva respectivamente.

- Las propiedades fisicoquímicas del aceite esencial de mastranto obtenidas experimentalmente fueron: densidad: 0,812 g/mL, pH: 4,87 e índice de refracción: 1,4772 Adim. esto indica la posible presencia de compuestos alifáticos oxigenados.

- El estudio de estabilidad muestra que las composiciones más adecuadas eran las de 1 y $5 \%$ de aceite esencial. Además, no presenta evidencia de contaminación microbiológica, cumpliéndose los parámetros que exigen las normas establecidas en los convenios internacionales para elaboración de cosméticos.

- Las pruebas dermatológicas no arrojaron anomalía ni se encuentra evidencia de que el producto final produzca efectos no deseados en la piel.

\section{REFERENCIAS}

Abagli, A. Z., y Alavo, T. B. C. (2011). Essential Oil from Bush Mint, Hyptis suaveolens, is as Effective as DEET for Personal Protection against Mosquito Bites. Open Entomology Journal, 5, 4548

Alcántara, B. (2015). Factibilidad técnicoeconómica y puesta en marcha de una planta tipo banco para la extración de aceite esencial de mastranto (hyptis saveoles) por arrastre con vapor. Universidad de Carabobo, ValenciaVenezuela 
Álvarez, D y Medina, N. (2008). Evaluación del proceso de extración de aceite esencial de mango empleando $\mathrm{CO} 2$ supercrítico. Universidad de Carabobo, Valencia

Bosch-Fusté, J., Riu-Aumatell, M., Guadayol, J. M., Caixach, J., López-Tamames, E., y Buxaderas, S. (2007). Volatile profiles of sparkling wines obtained by three extraction methods and gas chromatography-mass spectrometry (GC-MS) analysis. Food Chemistry, 105(1), 428-435

Castellanos Gómez, M. A. (2014). Determinación de los compuestos volátiles en pentacalia vaccinioides, su estudio antioxidante y antimicrobiano (Tesis de Maestría, Facultad de Ciencias de Pontificia Universidad Javeriana, Bogotá)

Conti, B., Benelli, G., Flamini, G., Cioni, P. L., Profeti, R., Ceccarini, L., y Canale, A. (2012). Larvicidal and repellent activity of Hyptis suaveolens (Lamiaceae) essential oil against the mosquito Aedes albopictus Skuse (Diptera: Culicidae). Parasitology research, 110(5), 2013-2021

Corbel, V., Stankiewicz, M., Pennetier, C., Fournier, D., Stojan, J., Girard, E., y Lapied, B. (2009). Evidence for inhibition of cholinesterases in insect and mammalian nervous systems by the insect repellent deet. BMC biology, $7(1), 47$

Chacín, J., Marquina, G., y Figueroa, Y. (2004). Extraction os mastranto (hyptis suaveolens) essencial oil using supercritical carbon dioxine. $V$ Encontro Brasileiro de fluidos supercríticos, Río de Janeiro

Daza, L. y Flores, N. (2006). Diseño de un repelente para insectos voladores con base a productos naturales. Universidad EAFIT, Medellín

Dutta, P. K., Tripathi, S., Mehrotra, G. K., y Dutta, J. (2009). Perspectives for chitosan based antimicrobial films in food applications. Food chemistry, 114(4), 1173-1182
Escobar, A. (2012). Diseño y puesta en marcha de una planta piloto de arrastre con vapor para la obtención de aceites esenciales. Universidad de Carabobo, Valencia- Venezuela

Guayadol Cunill, J. (1994). Estudio de los parámetros para la determinación de los compuestos orgánicos volátiles de la oleorresina de pimentón. Universidad Politécnica de Cataluña, Barcelona

ISO_ FDIS 21149 (2006). CosmeticsMicrobiology-Enumeration and detection of aerobic mesophilic bacteria. ISO

Khalid, S., Malik, A. U., Saleem, B. A., Khan, A. S., Khalid, M. S., y Amin, M. (2012). Tree age and canopy position affect rind quality, fruit quality and rind nutrient content of 'Kinnow'mandarin (Citrus nobilis Lourx Citrus deliciosa Tenora). Scientia Horticulturae, 135, 137-144

Messias, M. C. T. B., Menegatto, M. F. D. M., Prado, A. C. C., Santos, B. R. D., y Guimarães, M. F. M. (2015). Uso popular de plantas medicinais e perfil socioeconômico dos usuários: um estudo em área urbana em Ouro Preto, MG, Brasil, Rev. Bras. Pl. Med., Campinas, 17(4), 76-104

Nannapaneni, R., Chalova, V. I., Crandall, P. G., Ricke, S. C., Johnson, M. G., y O'Bryan, C. A. (2009). Campylobacter and Arcobacter species sensitivity to commercial orange oil fractions. International journal of food microbiology, 129(1), 43-49

Ostos, N., y Rodríguez,M. (2016). Elaboración de un repelente de insectos voladores a base de aceite esencial de mastranto (hiptis suaveolens) extraidos por diferentes me'ttodos. Universidad de Carabobo, Valencia-Venezuela

Prat,A., y Tort-Martorell, X., (2004). Métodos estadísticos: control y mejora de la calidad. Barcelona: Politecnos 1 
Peerzada, N. (1997). Chemical composition of the essential oil of Hyptis suaveolens. Molecules, 2(11), 165-168

Pérez, D., Marquina, G., y Alvarado, C. (2011). Evaluación de la composición química del aceite esencial de mastranto (hyptis suaveolens) según su origen geográfico en el estado Yaracuy, Venezuela. Revista Ingeniería y Sociedad UC. 7, (2), 95-105

Ranson, H., N'guessan, R., Lines, J., Moiroux, N., Nkuni, Z., y Corbel, V. (2011). Pyrethroid resistance in African anopheline mosquitoes: what are the implications for malaria control?. Trends in parasitology, 27(2), 91-98
Singh, H. P., Kaur, S., Negi, K., Kumari, S., Saini, V., Batish, D. R., y Kohli, R. K. (2012). Assessment of in vitro antioxidant activity of essential oil of Eucalyptus citriodora (lemon-scented Eucalypt; Myrtaceae) and its major constituents. LWT-Food science and Technology, 48(2), 237-241

Soto, L., Ojeda, L. Rojas, B., Sulbarán, J., Peña, M., Berrader, M., y Fernandez, V. (2013). Caracterización química del aceite esencial de toronja (citrus paradisi L.). Revista Facultad de Agronomía 30 (2), 266-283 